\title{
Clinical Features of Hereditary and Mast Cell-mediated Angioedema Focusing on the Differential Diagnosis in Japanese Patients
}

\author{
Isao Ohsawa ${ }^{1,2}$, Daisuke Honda ${ }^{2}$, Atsuko Hisada ${ }^{2,3}$, Hiroyuki Inoshita $^{2}$, \\ Kisara Onda-Tsueshita ${ }^{2}$, Satoshi Mano ${ }^{2}$, Nobuyuki Sato ${ }^{2,4}$, Yuya Nakamura ${ }^{1}$, \\ Tatsuo Shimizu ${ }^{1,5}$, Hiromichi Gotoh ${ }^{1}$, Yoshikazu Goto ${ }^{1}$, \\ Yusuke Suzuki $^{2}$ and Yasuhiko Tomino ${ }^{6}$
}

\begin{abstract}
:
Objective The present study was designed to identify the clinical characteristics that permit the differential diagnosis of hereditary angioedema (HAE) and mast cell-mediated angioedema (Mast-AE) during the first consultation.

Methods The medical histories and laboratory data of 46 patients with HAE and 41 patients with Mast-AE were compared.

Results The average age of onset in the HAE group (19.8 \pm 9.0 years) was significantly lower than that in the Mast-AE group (35.2 \pm 12.0 years). The incidence of familial angioedema (AE) in the HAE group (73.9\%) was significantly higher than that in the Mast-AE group (9.7\%). The frequency of history of AE in the extremities, larynx, or gastrointestinal tract was significantly higher in the HAE group. The frequency of $\mathrm{AE}$ episodes of the lips and eyelids was significantly lower in the HAE group. The serum C4 concentration and CH50 titer were lower than the normal limit in $91.3 \%$ and $45.6 \%$ of the patients in the HAE group, respectively; in Mast-AE group the serum C4 concentration and $\mathrm{CH} 50$ titer were significantly lower than the normal limit in $4.8 \%$ and $0 \%$ of the patients, the difference between the two groups was statistically significant. A C1-inhibitor (C1-INH) activity level of $<50 \%$ was observed in all of the HAE patients, but none of the Mast-AE patients. The mean serum IgE titer in the HAE group $(120.8 \pm 130.5 \mathrm{IU} / \mathrm{mL})$ was significantly lower than that in the Mast-AE group $(262.2 \pm 314.9 \mathrm{IU} / \mathrm{mL})$.

Conclusion The parameters within the patients' medical histories, such as the age at the onset of AE, a family history of $\mathrm{AE}$, and the locations of past $\mathrm{AE}$ episodes are critical for the successful diagnosis of the disease. Measurements of the C4 and C1-INH activity are very useful for differential diagnosis of HAE from Mast-AE.
\end{abstract}

Key words: hereditary angioedema, C1-inhibitor, C4, IgE, mast cell

(Intern Med 57: 319-324, 2018)

(DOI: 10.2169/internalmedicine.8624-16)

\section{Introduction}

Hereditary angioedema (HAE) is a rare and potentially life-threatening disease that results from mutations of the gene encoding $\mathrm{C} 1$-inhibitor (C1-INH). The excessive local formation of bradykinin due to the pathological activation of the factor XII-driven plasma contact system is consistently

\footnotetext{
${ }^{1}$ Nephrology Unit, Saiyu Soka Hospital, Japan, ${ }^{2}$ Division of Nephrology, Department of Internal Medicine, Juntendo University Faculty of Medicine, Japan, ${ }^{3}$ Department of Nephrology, Ikegami General Hospital, Japan, ${ }^{4}$ Deprtment of Internal Medicine, Niigata Rosai Hospital, Japan, ${ }^{5}$ Department of Internal Medicine, Saiyu Kawaguchi Clinic, Japan and ${ }^{6}$ Medical Corporation SHOWAKAI, Japan Received: November 29, 2016; Accepted: June 1, 2017; Advance Publication by J-STAGE: November 1, 2017 Correspondence to Dr. Isao Ohsawa, i.osawa@saiyukai.com
} 
found in patients with acute episodes of $\operatorname{HAE}(1,2)$. Because plasma-derived $\mathrm{C} 1$-INH concentrate is available for the first-line treatment of acute HAE attacks, an early diagnosis and prompt treatment are the keys to the best management and have improved patient outcomes. However, European reports indicate a delay between the onset of symptom and the diagnosis, with a mean diagnostic delay of almost 10-15 years (3-6). Our previous Japanese survey also confirmed that the time to the diagnosis (more than 13 years in some cases) was unacceptably long (7). These findings clearly highlight the urgent need for increasing awareness of the clinical symptoms of HAE and for educational campaigns aimed at medical staff in Japan.

Urticaria is a common disorder, which occurs in $20 \%$ of individuals at some point during their lives (8). Mast cells play a crucial role in urticaria, and in many cases of angioedema (AE). Mast cell degranulation elicits the release of various mediators, such as histamine, leukotrienes and prostaglandins. The majority of mast cell-mediated $\mathrm{AE}$ (Mast-AE) is controlled with the use of anti-histamines or corticosteroids. From the perspective of primary care, approximately $40 \%$ of patients with urticaria experience $\mathrm{AE}$ (8). On the other hand, $10 \%$ of patients have recurrent $\mathrm{AE}$, which is associated with mild urticaria (or the absence of urticaria) (9). Routine laboratory screening tests, independent of the patient's history and physical examination results should be discouraged, since they are of little value in discovering the trigger for Mast-AE reactions $(10,11)$. Since the true number of patients with Mast-AE might greatly exceed the number of HAE patients, it is crucial to make an accurate differential diagnosis. With this in mind, we aimed to clarify the clinical differences between HAE and MastAE.

\section{Materials and Methods}

Forty-six (M:F=16:30) HAE patients and 41 (M:F=14:27) Mast-AE patients who were treated at clinics specialized in HAE care at Juntendo University Hospital (Tokyo, Japan) and Saiyu Soka Hospital (Saitama, Japan), were enrolled. All of the patients had confirmed AE. The patients also had introduction letters from their general physicians or photographs of their own AE site(s).

HAE was diagnosed according to the diagnostic criteria described by Agostoni et al. (12) in addition to the Japanese guidelines published in 2010 (13). Within these guidelines, the major clinical criteria are: 1) self-limiting, noninflammatory subcutaneous AE without major urticarial rash, often recurrent and often lasting for more than 12 hours, 2) self-remitting abdominal pain without a clear organic etiology, often recurrent and often lasting for more than 6 hours, and 3) recurrent laryngeal edema. The minor clinical criteria are a family history of recurrent $\mathrm{AE}$ and/or abdominal pain and/or laryngeal edema. The laboratory criteria include: 1) two separate recordings of $\mathrm{C} 1$ inhibitor antigenic levels of $<50 \%$ the normal range in asymptomatic patients of 12 months of age or older who had not recently received a C1INH infusion, 2) two separate recordings of the $\mathrm{C} 1$ inhibitor functional levels of $<50 \%$ the normal range in asymptomatic patients of 12 months of age or older who had not recently received $\mathrm{C} 1-\mathrm{INH}$ infusion, and 3) a mutation in the $\mathrm{C} 1$ inhibitor gene affecting the synthesis and/or function of protein. Using this approach, HAE was diagnosed if there was evidence of one major (points 1-3 above) clinical criterion and one laboratory criterion. AE patients, with the exception of patients with HAE, drug-induced $\mathrm{AE}$ and $\mathrm{AE}$ with autoimmune disease, were enrolled as Mast-AE patients. We paid special attention to the past symptoms of acute or chronic urticaria, prodromal erythema marginatum, the effectiveness of anti-histamine drugs and/or corticosteroids, estrogen-induced $\mathrm{AE}$ and menstruation-related $\mathrm{AE}(2,9,11)$.

All of the historical physical locations of AE occurrences were recorded for each patient. Blood samples were obtained from the patients at the first consultation for a diagnosis of AE to be made under normal conditions (no swelling and no medication). The following laboratory data were collected: white blood cells (WBC), eosinophils (Eo), red blood cells (RBC), hemoglobin (Hb), hematocrit (Htc), serum creatinine $(\mathrm{Cr})$, total protein $(\mathrm{TP})$, albumin (Alb), immunoglobulin (Ig) G, IgM, IgA, and $\operatorname{IgE}, \mathrm{C} 3, \mathrm{C} 4$, total hemolytic complement activity $(\mathrm{CH} 50)$ and $\mathrm{C}$-reactive protein (CRP) were collected. For the differential diagnosis, the serum concentration of immune complex (IC) (determined using a C1q-binding assay), $\mathrm{Clq}$ and cryoglobulin were evaluated, and a genetic analysis of C1-INH was performed in some patients with low functional levels of $\mathrm{C} 1-\mathrm{INH}$ and with no family history of AE. The functional levels of C1INH were determined using a chromogenic assay (Sysmex, Kobe, Japan). For the differentiation of autoimmune disease, the serum anti-nuclear antibody (ANA) levels were measured. The study was conducted in accordance with the Declaration of Helsinki (1995) and was approved by the Institutional Review Board at Juntendo University and Saiyu Soka Hospital. Written informed consent was obtained from all of the participating patients.

The GraphPad Prism 5 for Windows software program (version 5.04; GraphPad, San Diego, USA) was used for the statistical analysis. Two-sided $\mathrm{p}$ values of $<0.05$ were considered to indicate statistical significance. Comparisons between two groups was performed using the Mann-Whitney $\mathrm{U}$ test. The relationship between categorical variables was analyzed using Fisher's exact test. All of the data were expressed as the mean \pm standard deviation (SD).

\section{Results}

The average age of onset in the HAE group (19.8 \pm 9.0 years) was significantly lower than that in the Mast-AE group $(35.2 \pm 12.0$ years) (Table 1$)$. Although the effectiveness of anti-histamine drugs and corticosteroids in treating AE attacks was high in the Mast-AE group, these drugs were also effective in a few HAE patients. We found the 
Table 1. Clinical Background.

\begin{tabular}{|c|c|c|c|c|}
\hline & & HAE & Mast-AE & $\mathrm{p}$ \\
\hline $\mathrm{n}$ & & 46 & 41 & \\
\hline Male:Female & & $16: 30$ & $14: 27$ & $\mathrm{~ns}^{* *}$ \\
\hline Age & & $41.7 \pm 13.3$ & $40.6 \pm 12.7$ & $\mathrm{~ns}^{*}$ \\
\hline \multicolumn{5}{|l|}{ Angioedema } \\
\hline Age of onset & & $19.8 \pm 9.0$ & $35.2 \pm 12.0$ & $\mathrm{p}<0.01 *$ \\
\hline Predromal erthema marginatum & (yes:no) & $5: 41$ & $0: 41$ & $\mathrm{~ns}^{* *}$ \\
\hline Effectiveness of anti-histamin drugs & (yes/no) & $3 / 16$ & $28 / 2$ & $\mathrm{p}<0.01 * *$ \\
\hline Effectiveness of corticosteroids & (yes/no) & $4 / 11$ & $10 / 1$ & $\mathrm{p}<0.01 * *$ \\
\hline Estrogen induced (if female) & (yes/no) & $4 / 26$ & $0 / 27$ & $\mathrm{~ns}^{* *}$ \\
\hline Menstruation-related (if female) & (yes/no) & $3 / 27$ & $0 / 27$ & $\mathrm{~ns}^{* *}$ \\
\hline \multicolumn{5}{|l|}{ Other medical histories } \\
\hline Acute or chronic urticaria & (yes:no) & 9:37 & 27:14 & $\mathrm{p}<0.01 * *$ \\
\hline Atopic dermatitis & (yes:no) & $4: 42$ & 2:39 & $\mathrm{ns}^{* *}$ \\
\hline Bronchial asthma & (yes:no) & $1: 45$ & $9: 32$ & $\mathrm{p}<0.01 * *$ \\
\hline Pollinosis & (yes:no) & $9: 37$ & $8: 33$ & $\mathrm{~ns}^{* *}$ \\
\hline Family history of AE & (yes:no) & $34: 12$ & $4: 37$ & $\mathrm{p}<0.01 * *$ \\
\hline
\end{tabular}

Table 2. The Number of Patients Having Former Subcutaneous Angioedema.

\begin{tabular}{lcccccc}
\hline & Extremities & Face & Lip & Eye lids & Buttocks & Genital \\
\hline HAE, $\mathrm{n}(\%)$ & $37(80.4)$ & $18(39.1)$ & $13(28.2)$ & $3(6.5)$ & $5(10.8)$ & $5(10.8)$ \\
Mast-AE, $\mathrm{n}(\%)$ & $4(9.7)$ & $8(19.5)$ & $23(56.0)$ & $17(41.4)$ & $1(2.4)$ & $0(0.0)$ \\
\hline \multicolumn{1}{c}{$\mathrm{p}$} & $<0.01$ & $\mathrm{~ns}$ & $<0.01$ & $<0.01$ & $\mathrm{~ns}$ & $\mathrm{~ns}$ \\
\hline
\end{tabular}

Fisher's exact test

ns: not significant

characteristic information about estrogen-induced $\mathrm{AE}$ and menstruation-related $\mathrm{AE}$ in female $\mathrm{HAE}$ patients but there were no statistically significant differences between the HAE and Mast-AE groups. The patients other allergic history (i.e., acute or chronic urticaria and bronchial asthma) were found significantly more frequently in the Mast-AE group. A family history of AE was noted in $73.9 \%$ of the patients in the HAE group; the frequency was significantly higher than that in the Mast-AE group (9.7\%).

For the analysis of the patients with former AE, the patient population was divided into two parts, patients with subcutaneous AE (Table 2) and patients with submucosal AE (Table 3). Among the subcutaneous cases, AE episodes were observed at the extremities (Fig. 1a) significantly more frequently in the HAE group (80.4\%) in comparison to the Mast-AE group (9.7\%). AE episodes involving the lips and eyelids were observed significantly less frequently in the HAE group $(28.2 \%, 6.5 \%)$ than in the Mast-AE group (56.0\%, 41.4\%). Genital AE was only observed in the HAE group ( 5 cases); however, the difference between the groups was not statistically significant. With respect to submucosal
$\mathrm{AE}$, the incidence of laryngeal AE episodes in the HAE group $(39.1 \%)$ was significantly higher than that in the Mast-AE group (17.0\%). Cases of upper air way obstruction (choking) were exclusively observed in the HAE group (6 cases). Three of the six cases had experienced suffocation and required emergency tracheotomy. The frequency of abdominal pain derived from gastrointestinal AE (Fig. 1b) was significantly higher in the HAE group $(56.5 \%)$ than in the Mast-AE group (12.1\%).

There were no significant differences in the WBC, Eo, $\mathrm{RBC}, \mathrm{Hb}, \mathrm{Htc}, \mathrm{Cr}$, TP, Alb, IgG, IgM, IgA, and CRP values of the HAE and Mast-AE groups. The mean IgE titer of the HAE group $(120.8 \pm 130.5 \mathrm{IU} / \mathrm{mL})$ was significantly lower than that of the Mast-AE group $(262.2 \pm 314.9 \mathrm{IU} / \mathrm{mL})$ (Table 4). The distribution of the $\mathrm{C} 3, \mathrm{C} 4, \mathrm{CH} 50$, and $\mathrm{C} 1-\mathrm{INH}$ activity levels is shown in Fig. 2. The mean serum C3 concentration of the HAE group and Mast-AE groups (104.4 \pm $23.7 \mathrm{mg} / \mathrm{dL}$ and $100.0 \pm 17.7 \mathrm{mg} / \mathrm{dL}$, respectively) was similar. A serum C4 concentration of less than normal limit $(<14$ $\mathrm{mg} / \mathrm{dL}$ ) was seen in $91.3 \%$ of the HAE patients and in $4.8 \%$ of the Mast-AE patients (Fisher's exact test, $\mathrm{p}<0.01$ ). In the 
Table 3. the Number of Patients Having Former Mucosal Angioedema and Symptoms.

\begin{tabular}{lcccc}
\hline & Oral cavity & Laryngeal & Choking & Abdominal pain \\
\hline HAE, $\mathrm{n}(\%)$ & $4(8.6)$ & $18(39.1)$ & $6(8.6)$ & $26(56.5)$ \\
Mast-AE, $\mathrm{n}(\%)$ & $5(12.1)$ & $7(17.0)$ & $0(0.0)$ & $5(12.1)$ \\
\hline $\mathrm{p}$ & $\mathrm{ns}$ & $<0.05$ & $<0.05$ & $<0.01$ \\
\hline
\end{tabular}

Fisher's exact test

ns: not significant
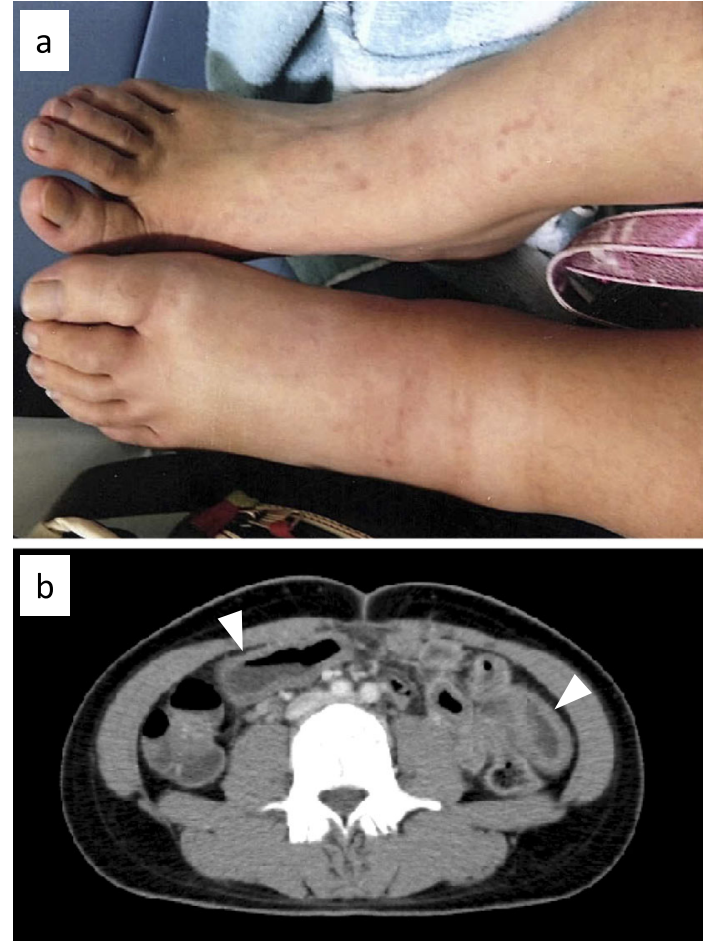

Figure 1. Pictures of angioedema from patients with hereditary angioedema. a: A 38-year-old female patient. Marked swelling of the left lower limb was observed. b: A 19-year-old female patient. Enhanced computed tomography of the abdomen demonstrated an expanded edematous small intestine (arrow head) and the accumulation of intestinal fluid.

HAE group, 9 of the 46 patients presented with serum levels of $\mathrm{C} 4$, which were below the lower limit of quantitation $(<2$ $\mathrm{mg} / \mathrm{dL}$ ); this was not observed in the Mast-AE group. A $\mathrm{CH}$ 50 titer below the normal limit $(<25 \mathrm{U} / \mathrm{mL})$ was seen in $45.6 \%$ of the HAE patients but not in the Mast-AE patients (Fisher's exact test, $\mathrm{p}<0.01$ ). In the HAE group, 6 of the 46 patients presented with $\mathrm{CH} 50$ titers that were below the lower limit of quantitation $(<7 \mathrm{U} / \mathrm{mL})$; this was not the case in the Mast-AE group. A C1-INH activity level of $<50 \%$ was observed in all $(100 \%)$ of the HAE patients but none of the Mast-AE patients (Fisher's exact test, $\mathrm{p}<0.01$ ). In the HAE group, 35 of 46 patients presented with an extremely low C1-INH activity level, which was below the lower limit of quantitation $(<25 \%)$.

\section{Discussion}

HAE is a potentially life-threatening disease caused by mutations in the SERPING1 gene, which results in the decreased synthesis (type I) or function (type II) of C1INH $(1,2)$. The prevalence of HAE is estimated to be 1 case per 50,000-100,000 people, with no reported bias among different ethnic groups (2). It is assumed that there are 2,000 to 3,000 HAE patients in Japan; however, based on the unofficial data collated from case reports in Japan and from several pharmaceutical company databases, it is estimated that 450 patients have been diagnosed in Japan.

The ability to differentially diagnose a patient during the first visit to an outpatient clinic is paramount. Although $\mathrm{AE}$ is associated with familiar symptoms, HAE patients typically wait years before being correctly diagnosed $(7,14)$. Because of the low awareness of HAE among physicians, the correct diagnosis of the disease is complicated by misdiagnosis with the dominant type of AE (Mast-AE). Thus, it is critical to educate doctors and/or medical staff on the specific complaints associated with HAE.

Our results highlight the importance of historical medical data, including the age of AE onset, a family history of AE, and the locations of past $\mathrm{AE}$ episodes. With regard to the average age of the patients when the first AE attack, the patients in the HAE group were significantly younger (in adolescence) in comparison to the Mast-AE group. Since our outpatient clinics are for adults, our data may be slightly skewed towards a higher age. In our study, anti-histamine drugs and corticosteroids showed significant efficacy in the Mast-AE group, but were only effective in 9 of 46 patients with HAE combined with acute or chronic urticaria. Thus, the effectiveness of these drugs cannot be used to reliably differentiate between HAE and Mast-AE.

Because an estrogen-responsive element in the promotor region of the factor XII gene is responsible for the exacerbation of $\mathrm{AE}(2,9)$, we only found a history of estrogeninduced $\mathrm{AE}$ and menstruation-related $\mathrm{AE}$ among the HAE patients.

As previously reported, $75 \%$ of HAE patients present with an autosomal dominant inheritance form of disease and $25 \%$ of HAE patients present with de novo mutations $(1,2)$. Because our data confirmed the high frequency of autosomal dominant inheritance $(73.9 \%)$, we can infer that monitoring the family history of AE would be very informative. Hun- 
Table 4. Laboratory Data Obtained at the First Consultation.

\begin{tabular}{llrrr}
\hline & & \multicolumn{1}{c}{ HAE } & \multicolumn{1}{c}{ Mast-AE } & $\mathrm{p}$ \\
\hline Eosinocyte $(/ \mu \mathrm{L})$ & & $191.3 \pm 267.0$ & $206.3 \pm 223.5$ & $\mathrm{~ns}$ \\
IgE $(\mathrm{IU} / \mathrm{mL})$ & & $120.8 \pm 130.5$ & $262.2 \pm 314.9$ & $\mathrm{p}<0.01^{*}$ \\
$\mathrm{C} 3(\mathrm{mg} / \mathrm{dL})$ & & $104.4 \pm 23.7$ & $100.0 \pm 17.7$ & $\mathrm{~ns}$ \\
$\mathrm{C} 4<14(\mathrm{mg} / \mathrm{dL})$ & (yes:no) & $42: 4$ & $2: 39$ & $\mathrm{p}<0.01^{* *}$ \\
CH50 $<25(\mathrm{U} / \mathrm{mL})$ & (yes:no) & $21: 25$ & $0: 41$ & $\mathrm{p}<0.01^{* *}$ \\
C1-INH activity $<50(\%)$ & (yes:no) & $46: 0$ & $0: 41$ & $\mathrm{p}<0.01^{* *}$ \\
\hline
\end{tabular}

*Mann-Whitney's U test

**Fisher's exact test

ns: not significant
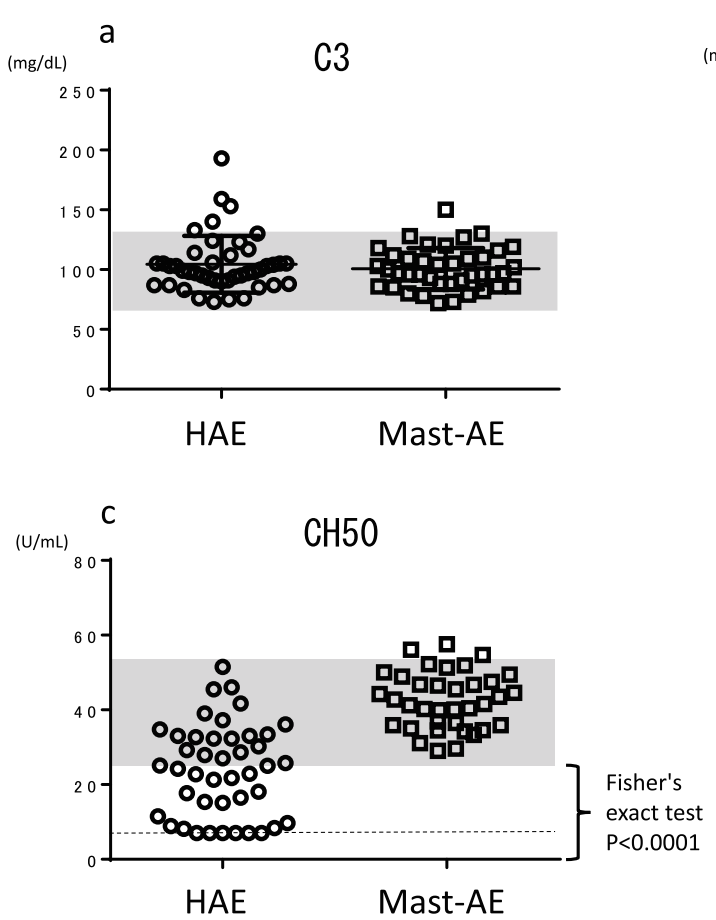
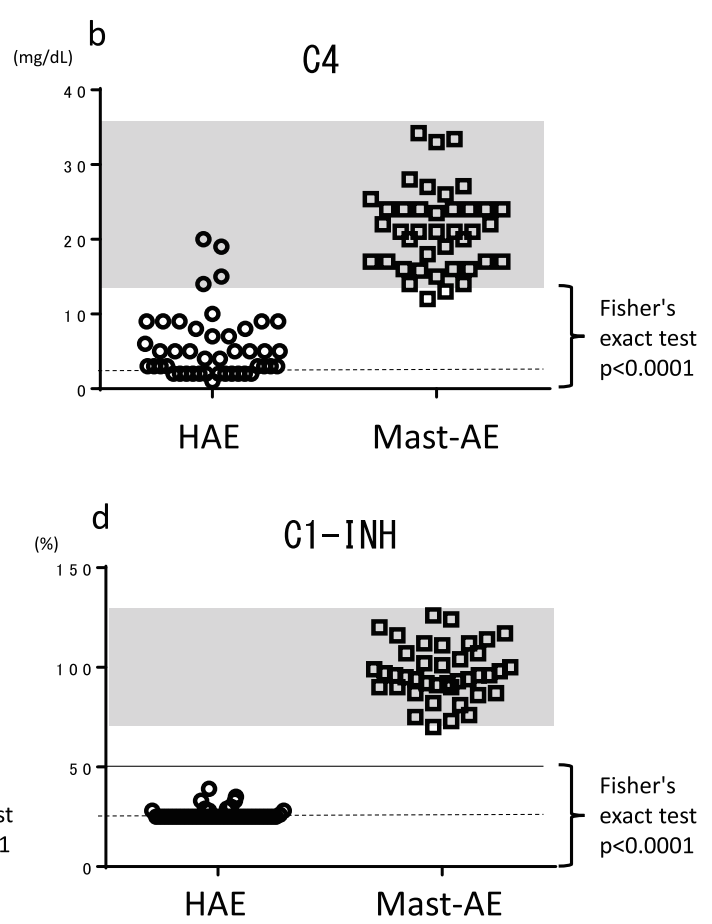

Figure 2. Dot plots of the complement system parameters. a: The serum levels of $\mathrm{C} 3$. b: The serum levels of C4. c: The serum titers of CH50. d: The serum activities of C1-inhibitor. The gray zone represents the normal range. The horizontal broken line represents the limit of quantitation. The horizontal line of Fig. 2d indicates a C1-inhibitor activity level of $50 \%$, which is stated in the diagnostic criteria (12). HAE: hereditary angioedema, Mast-AE: mast cell-mediated angioedema, C1-INH: C1inhibitor

dreds of different heterozygous mutations of $\mathrm{C} 1$-INH have been described in a database (HAEdb, http://hae.enzim.hu), Japanese investigators are now going to perform a genetic analysis of a nationwide registration system that was established by The Japanese Association for Complement Research.

The AE attacks occurred in characteristic locations in both groups. In the HAE group, a history of AE in the extremities was the most common location of subcutaneous $\mathrm{AE}(80.4 \%)$. A previous per-patient analysis also reported that the extremities were the most affected parts in subcutaneous skin lesions among 209 HAE patients (15). The lips and eyelids were a common location in the Mast-AE group, which is consistent with mast cell degranulation being triggered by an antigen that enters these anatomical sites. On the other hand, mucosal AE was not common in the MastAE group. Strikingly, the HAE patients developed lifethreatening laryngeal edema and gastrointestinal AE. These severe attacks clearly pose obstacles in the daily lives of patients. In the future, we therefore need to understand the burden of illness in HAE patents with objective evaluation methods, such as the health quality of life (HRQoL) and the Dermatology Life Quality Index (DLQI) (16).

The clinical guidelines of the World Allergy Organization (WAO) recommend the measurement of the serum $\mathrm{C} 4$ level as the best specific screening test. The detection of $\mathrm{C} 4$ concentrations below the normal range shows $98 \%$ specificity in the diagnosis of HAE (2). Although our results showed that the specificity for a diagnosis of HAE was slightly lower (42 of 46 patients, 91.3\%), the serum $\mathrm{C} 4$ level in $4 \mathrm{HAE}$ 
patients with a normal basal concentration did drop below the normal limit during AE attacks. Two of forty-one MastAE patients presented with low serum C4 levels, but had normal levels of immunoglobulins and CRP, and had no signs of autoimmune disease. Such patients may have genetic alterations at the $\mathrm{C} 4$ locus that disrupt their protein function (17). Titers of CH50 that were below the normal range were only observed in the HAE group but showed low diagnostic sensitivity $(45.6 \%)$. In contrast to the measurement of $\mathrm{CH} 50$, a low $\mathrm{C} 1$-INH activity $(<50 \%$ of normal) was observed in all of the patients in the HAE group and showed $100 \%$ specificity in the diagnosis of HAE. None of the patients in the Mast-AE group presented with low C1INH activity. In our country, the measurement of the antigenic C1-INH levels is not currently approved by the Japanese health insurance system; thus, we measured the antigenic C1-INH levels at the patient's or research institution's expense, in some cases. Our results clearly indicated that the measurement of the C1-INH activity would be sufficient for making a diagnosis of HAE.

In conclusion, the characteristic features of AE-related medical history combined with the results of complement tests in HAE patients are highly informative when examined at the first consultation.

The authors state that they have no Conflict of Interest (COI).

\section{Financial Support}

This work was supported by JSPS KAKENHI Grant Number 26461493 and 26460654.

\section{Acknowledgement}

We wish to thank my colleagues in the Division of Nephrology, Department of Internal medicine, Juntendo University Faculty of Medicine, Tokyo, Japan and Nephrology unit, Saiyu Soka Hospital, Saitama, Japan

\section{References}

1. Zuraw BL. Hereditary angioedema. N Engl J Med 359: 10271036, 2008.

2. Craig T, Pürsün EA, Bork K, et al. WAO guideline for the management of hereditary angioedema. World Allergy Organ J 5: 182199, 2012.

3. Bygum A. Hereditary angio-oedema in Denmark: a nationwide survey. Br J Dermatol 161: 1153-1158, 2009.
4. Psarros F, Koutsostathis N, Farmaki E, Speletas MG, Germenis AE. Hereditary angioedema in Greece: the first results of the Greek hereditary angioedema registry. Int Arch Allergy Immunol 164: 326-332, 2014.

5. Roche O, Blanch A, Caballero T, Sastre N, Callejo D, López-Trascasa M. Hereditary angioedema due to $\mathrm{C} 1$ inhibitor deficiency: patient registry and approach to the prevalence in Spain. Ann Allergy Asthma Immunol 94: 498-503, 2005.

6. Jolles S, Williams P, Carne E, et al. A UK national audit of hereditary and acquired angioedema. Clin Exp Immunol 175: 59-67, 2014.

7. Ohsawa I, Honda D, Nagamachi S, et al. Clinical manifestations, diagnosis, and treatment of hereditary angioedema: survey data from 94 physicians in Japan. Ann Allergy Asthma Immunol 114: 492-498, 2015

8. Amar SM, Dreskin SC. Urticaria. Prim Care 35: 141-157, vii-viii, 2008.

9. Bork K. Angioedema. Immunol Allergy Clin North Am 34: 23-31, 2014.

10. Kozel MM, Bossuyt PM, Mekkes JR, Bos JD. Laboratory tests and identified diagnoses in patients with physical and chronic urticaria and angioedema: a systematic review. J Am Acad Dermatol 48: 409-416, 2003.

11. Lang DM, Aberer W, Bernstein JA, et al. International consensus on hereditary and acquired angioedema. Ann Allergy Asthma Immunol 109: 395-402, 2012.

12. Agostoni A, Aygören-Pürsün E, Binkley KE, et al. Hereditary and acquired angioedema: problems and progress: proceedings of the third $\mathrm{C} 1$ esterase inhibitor deficiency workshop and beyond. J Allergy Clin Immunol 114: S51-S131, 2004.

13. Horiuchi T, Ohi H, Ohsawa I, et al. Guideline for hereditary angioedema (HAE) 2010 by the Japanese Association for Complement Research - secondary publication. Allergol Int 61: 559-562, 2012.

14. Zanichelli A, Magerl M, Longhurst H, Fabien V, Maurer M. Hereditary angioedema with $\mathrm{C} 1$ inhibitor deficiency: delay in diagnosis in Europe. Allergy Asthma Clin Immunol 9: 29, 2013.

15. Bork K, Meng G, Staubach P, Hardt J. Hereditary angioedema: new findings concerning symptoms, affected organs, and course. Am J Med 119: 267-274, 2006.

16. Bygum A, Aygören-Pürsün E, Caballero T, et al. The hereditary angioedema burden of illness study in Europe (HAE-BOISEurope): background and methodology. BMC Dermatol 12: 4, 2012.

17. Hauptmann G, Goetz J, Uring-Lambert B, Grosshans E. Component deficiencies: 2. The fourth component. Prog Allergy 39: 232$249,1986$.

The Internal Medicine is an Open Access article distributed under the Creative Commons Attribution-NonCommercial-NoDerivatives 4.0 International License. To view the details of this license, please visit (https://creativecommons.org/licenses/ by-nc-nd/4.0/).

\section{(C) 2018 The Japanese Society of Internal Medicine} Intern Med 57: 319-324, 2018 